\title{
Landsat8 Satellite Image Classification with ERDAS for Mapping the Kalambatritra Special Reserve
}

\author{
Arisetra Razafinimaro ${ }^{1,2,3,}$, , Aimé Richard Hajalalaina ${ }^{1,2,3}$, ZoJaona Tantely Reziky ${ }^{4}$, \\ Eric Delaitre ${ }^{5}$, Avisoa Andrianarivo ${ }^{6}$ \\ ${ }^{1}$ Department of Informatics, School of Management and Technological Innovation, Fianarantsoa, Madagascar \\ ${ }^{2}$ Laboratory of Computer Science and Mathematics Applied to Development, Fianarantsoa University, Fianarantsoa, Madagascar \\ ${ }^{3}$ Informatics-Geomatics Mathematics and Applications, Fianarantsoa University, Fianarantsoa, Madagascar \\ ${ }^{4}$ Higher Polytechnic School of Antananarivo, Antananarivo University, Antananarivo, Madagascar \\ ${ }^{5}$ Research Institute for Development, Montpellier, France \\ ${ }^{6}$ Department of Geography, Antananarivo University, Antananarivo, Madagascar
}

Email address:

tokyarisetra@gmail.com (A. Razafinimaro), arhajalalaina@yahoo.fr (A. R. Hajalalaina), zojaona@yahoo.fr (Z. T. Reziky), eric.delaitre@ird.fr (E. Delaitre)

${ }^{*}$ Corresponding author

\section{To cite this article:}

Arisetra Razafinimaro, Aimé Richard Hajalalaina, ZoJaona Tantely Reziky, Eric Delaitre, Avisoa Andrianarivo. Landsat8 Satellite Image Classification with ERDAS for Mapping the Kalambatritra Special Reserve. American Journal of Remote Sensing.

Vol. 9, No. 1, 2020, pp. 16-22. doi: 10.11648/j.ajrs.20210901.12

Received: December 17, 2020; Accepted: December 24, 2020; Published: February 23, 2021

\begin{abstract}
This paper focuses on the Landsat 8 satellite image classification of the OLI sensor via the remote sensing software Erdas Imagine in order to calculate the land cover surface and to establish the mapping of the special reserve Kalambatritra of Madagascar for the year 2018. For this, we adopted the methodology of satellite image processing based on supervised classification algorithms. The processing was moved to spectral preparation and improvement of spatial resolution using the blue, green, red, near infrared and panchromatic channels. Then, a comparison study of the supervised classification algorithms was done to obtain a more accurate result. The validation of the classification results was performed using several reference points, a previous national processing result already validated in the field and the Google earth image of the same year. After repeating the classification several times, we obtained accuracies of $77 \%, 75 \%, 88 \%, 84 \%$ and $90 \%$ with Kappa indices of $0.64,0.61,0.80,0.76$ and 0.84 for the Spectral Angle Mapper, Spectral Correlation Mapper, Maximum Likelihood, Mahalanobis Distance and Minimum Distance. Based on these results, the minimum distance showed a higher accuracy and gave us 13462.1842 ha of forest area, 16798.8006 ha of prairie for the year 2018.
\end{abstract}

Keywords: Image Processing, Landsat8, Forest Area Kalambatritra, Erdas Imagine, Land Use, Classification

\section{Introduction}

Supervised classification is a very important means in satellite image processing for mapping land use and determining forest area. For this purpose, images from SPOT, Landsat, Terra, Sentinel satellites are useful and allow this work because they are rich in spectral and spatial information at the same time. Thus, several scientific works have carried out experiments on these images such as the article used Landsat 7 ETM+ image to detect a wetland in Malagasy forest [1], and used SPOT images to detect land use changes in the humid forest [2]. Our study continues their work but we are focusing on the study of low altitude dense humid forest using Landsat 8 image from the OLI sensor.

Indeed, our Kalambatritra study area is located in the South-East zone of Madagascar. It is characterized as low altitude dense forest. This zone is often victim to human acts such as charcoal, Tavy cultivation, bushfires. These acts lead to the reduction of the forest surface of this special reserve. The classification facilitates the calculation of this area. However, each classification method gives different results [3-5].

In this article, we carry out the treatments with the ERDAS imagine. This software proposes supervised classification algorithms. Thus, the treatments are carried out in three phases. 
They include pre-treatment, classification and post-classification. The validation of the result is done by the image of Google earth at the same date and a national result already validated. The accuracy of the result obtained defines whether or not the processing is repeated. Thus, the results of our experiments show that the precision can be higher with the algorithm of minimum distance with a Kappa index of 0.84 .

\section{Presentation of the Study Area}

The Special Reserve of Kalambatritra was created by the decree $n^{\circ} 5959$ of April 24th, 1959 and is located in the South East of Madagascar ( $23^{\circ} 28$ South and $46^{\circ} 29$ East), at the limit of the regions of Ihorombe and Anosy, $55 \mathrm{~km}$ East of Betroka. This zone includes in the Path "159" and Row "076" of Landsat. Kalambatritra has an altitude of 1300 to $1500 \mathrm{~m}$. Figure 1 shows us the limit of the Kalambatritra Special Reserve.

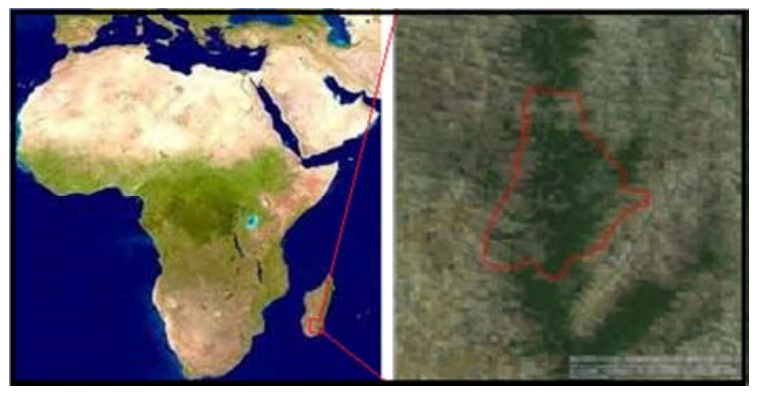

Figure 1. Limit of the special reserve Kalambatritra.

\section{Presentation of Data Used}

In this study, we need a satellite image that we choose from the Landsat 8 satellite image year 2018 because this image is free of charge and its characteristic is highly suitable for forest area classification. Landsat 8 images can be downloaded from the link http://www.googleearth.gov. An image from this satellite is made up of 11 channels but we are interested in band 2, band 3 , band 4 , band 5 and band 8 (panchromatic) from the OLI sensor. Table 1 shows the characteristics of these bands.

Table 1. Feature four bands and the Panchromatic band of Landsat 8 OLI sensor.

\begin{tabular}{llll}
\hline Band & Wavelength & resolution & Color \\
\hline 2 & $0,450-0,515 \mu \mathrm{m}$ & $30 \mathrm{~m}$ & Blue \\
3 & $0,525-0,600 \mu \mathrm{m}$ & $30 \mathrm{~m}$ & Green \\
4 & $0,630-0,680 \mu \mathrm{m}$ & $30 \mathrm{~m}$ & Red \\
5 & $0,845-0,885 \mu \mathrm{m}$ & $30 \mathrm{~m}$ & Near Infrared \\
8 & $0,500-0,680 \mu \mathrm{m}$ & $15 \mathrm{~m}$ & Panchromatic \\
\hline
\end{tabular}

\section{Methodology on the Determination of Land Use}

In order to conduct this study properly, we are joining a rather generic method in satellite image processing using supervised classification. This method involves pre-processing, classification and post-classification $[6,2]$. Figure 2 shows the steps in this method.
Concatenation: This step allows us to combine the spectral bands (B2, B3, B4 and B5) to form a multispectral Landsat 8 image.

Fusion: The panchromatic band of the OLI sensor (B8) has a resolution of $15 \mathrm{~m}$. Fusion using this band can improve the resolution of our multispectral image. In this step, we have the previous result and the panchromatic band as input data and a $15 \mathrm{~m}$ multispectral image as output.

Color composition: This step allows us to distinguish objects in true color or false color mode based on RGB (Red-Green-Blue). It makes it easy to perform the visual analysis necessary for classification.

Image classification: In this step, we compare the five classification algorithms supervised under ERDAS Imagine to define the best classifier for Kalambatritra.

The Spectral Angle Mapper [7-11] is a classifier that determines the spectral similarity between two spectra by calculating the spectral angle between the spectral signatures of the image pixels and the spectral signatures of the training data. In this, the SAM treats them as vectors in a space of a dimension equal to the number of bands. Furthermore, the length of the vector represents the brightness of the pixel while the direction represents the spectral characteristic of the pixel. The variation in illumination mainly affects the changes in vector length, while the spectral variability between different spectra affects the angle between their corresponding vectors.

$$
\theta=\cos ^{-1}\left(\frac{\sum_{i=1}^{n} a_{i} b_{i}}{\sqrt{\sum_{i=1}^{n} a_{i}^{2} \sum_{i=1}^{n} b_{i}^{2}}}\right)
$$

Hence a: the spectral signature of the image pixel $b$ : the spectral signature of the training data and $n$ is the number of spectral bands in the image.

The Spectral Correlation Mapper or SCM [12, 13] is an improvement of the SAM algorithm. This supervised classification algorithm is based on a comparison of the spectral image with a reference spectrum. This reference spectrum is user-defined. In this, the comparison is performed through a similarity criterion.

$$
\mathrm{SCM}=\frac{\sum_{i=1}^{n}\left(a_{i}-\bar{a}\right)\left(b_{i}-\bar{b}\right)}{\sqrt{\sum_{i=1}^{n}\left(a_{i}-\bar{a}\right)^{2}} \sum_{i=1}^{n}\left(b_{i}-\bar{b}\right)^{2}}
$$

Hence a: the spectral signature of the image pixel $b$ : the spectral signature of the training data and $n$ is the number of spectral bands in the image.

Maximum likelihood [14-16] is one of the best known supervised classification methods used in the world of satellite image processing. This method is based on the probability that a pixel belongs to a particular class. That is, a pixel is classified in the corresponding class when it has the maximum probability for that class. However, it uses the probability function as the decision rule used. In other words, the maximum likelihood classifier quantitatively evaluates the variance and covariance of spectral response models when classifying an unknown pixel. 


$$
\hat{p}\left(x \mid w_{i}\right)=\frac{1}{(2 \pi)^{\frac{1}{2}} \widehat{\sigma}_{i}} \exp \left[-\frac{1}{2} \frac{\left(x-\widehat{\mu}_{i}\right)^{2}}{\widehat{\sigma}_{i}^{2}}\right]
$$

Hence $\mathrm{x}$ : value of the brightness of the $\mathrm{X}$ axis

$\hat{\mu}_{i}$ : average class value

$\hat{\sigma}_{i}$ : measurement of class variance

The Mahalanobis distance [17] is a measure of the distance between two points in space defined by at least two correlated variables. This classifier takes into account class variability. That is, it uses the statistic for each class based on a covariance matrix. It is similar to the minimum distance classifier for the mode of classifying data into different classes except that it uses a covariance matrix. The mathematical function that can calculate the Mahalanobis distance is presented as follows [18].

$$
M_{h}^{2}=\frac{1}{2}\left(x-m_{i}\right)^{t} C_{i}^{-1}\left(x-m_{i}\right)
$$

Hence:

$M_{h}^{2}:$ the distance of mahalanobis

$\left|C_{i}\right|$ : Determinant of the covariance matrix of class $i$ $\mathrm{x}$ :matrix of dimension $\mathrm{n}$ of a pixel $m_{i}:$ mean vector $\mathrm{t}$ : transposed from the matrix base

The minimum distance [19] is used to classify remote sensing data into different classes. It requires finding the mean value of each class called a centroid and then calculating the Euclidean distance between the mean value of the classes and the unknown value of the image pixel. Each pixel will be assigned to the corresponding classes according to the shortest distance between them. The implementation of this classifier is computationally simple and easy to use. The following formula can calculate the Euclidean distance between two points [16].

$$
\text { Dist }=\sqrt{\left(B V_{i j k}-\mu_{c k}\right)^{2}+\left(B V_{i j l}-\mu_{c l}\right)^{2}}
$$

Hence: $\mu_{c k}$ and $\mu_{c l}$ are the mean of the vectors in class $\mathrm{c}$ measured from the band $\mathrm{k}$ and 1 .

The validations of our results are done using the image of Google earth and a result of image processing (national) of Madagascar already validated. In this, we use one hundred (100) validation points for each result of the classification.

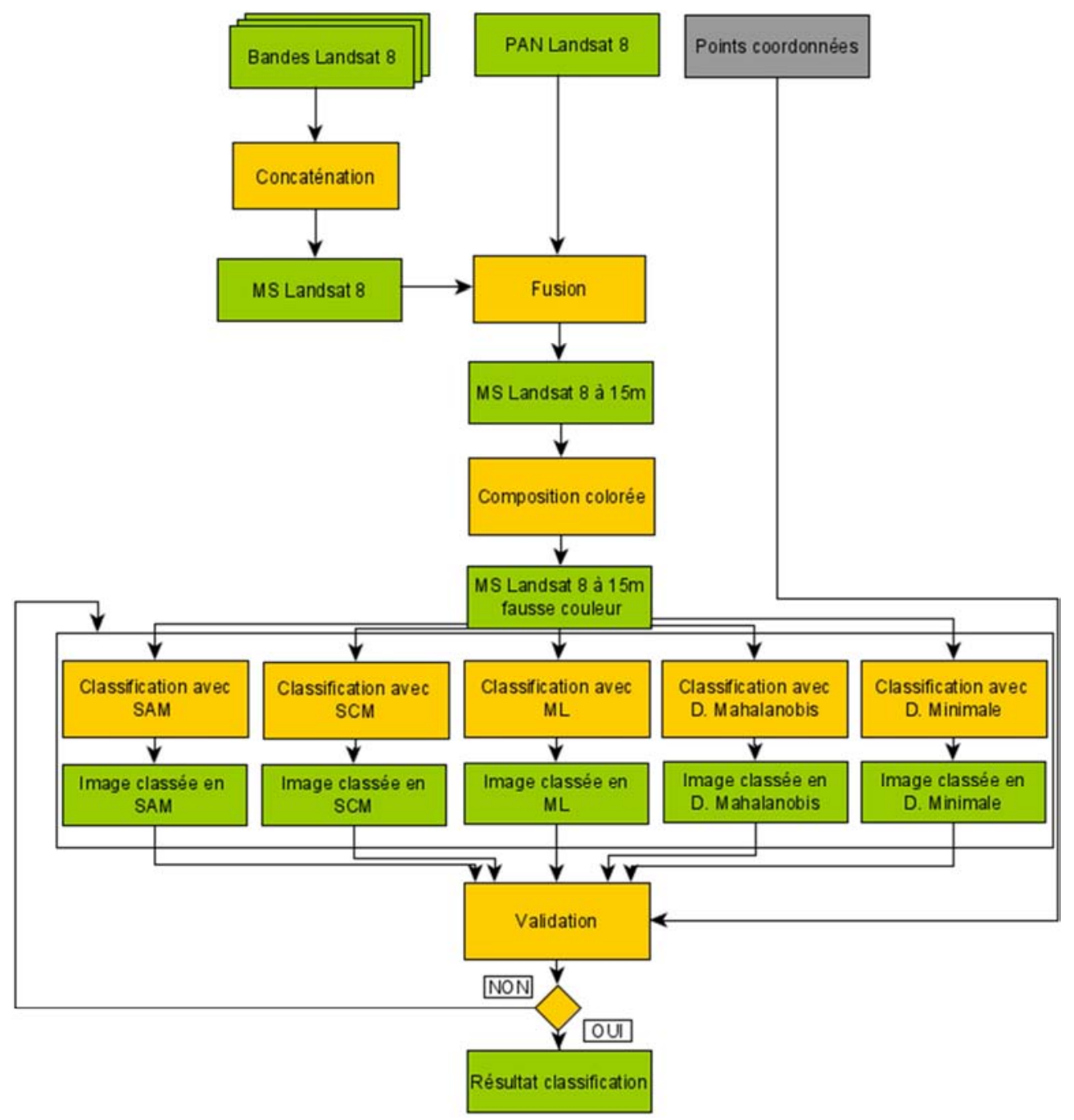

Figure 2. Methodology for the determination of land use. 


\section{Experimentations}

Using the ERDAS "stack" function, we obtained a multispectral image of four spectral bands with a resolution of $30 \mathrm{~m}$. Figure 3 illustrates our multispectral image in true color mode and in false color mode.

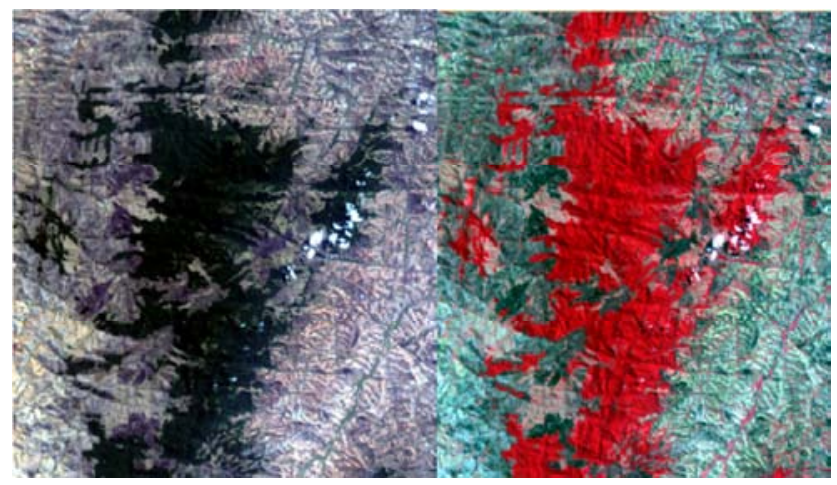

Figure 3. Landsat true color and false color multispectral image of Kalambatritra year 2018

Figure 4 shows the result of merging our Landsat 8 multispectral image with the panchromatic band at $15 \mathrm{~m}$ resolution.

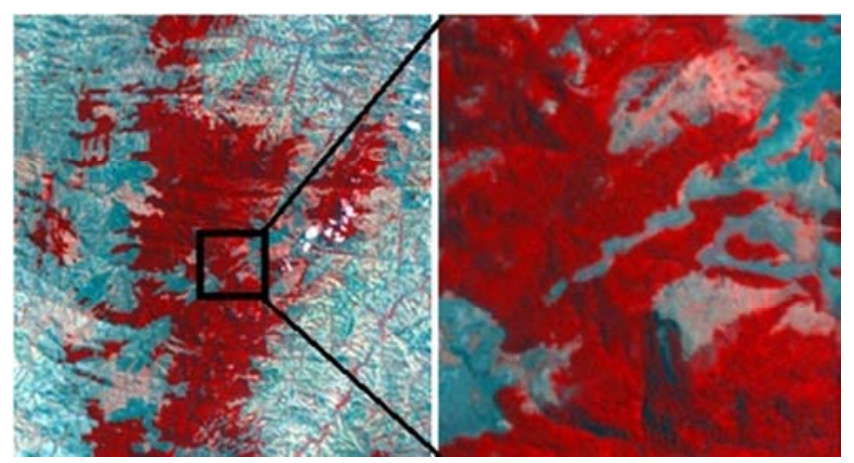

Figure 4. Multispectral Landsat 8 to $15 \mathrm{~m}$ resolution image of Kalambatritra year 2018 .

After repeating the classification several times, we obtained the following results.

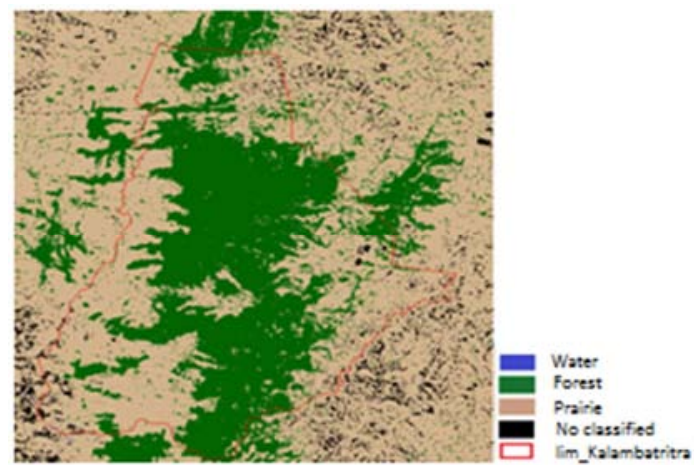

Figure 5. Result of the Kalambatritra classification with the SAM algorithm.

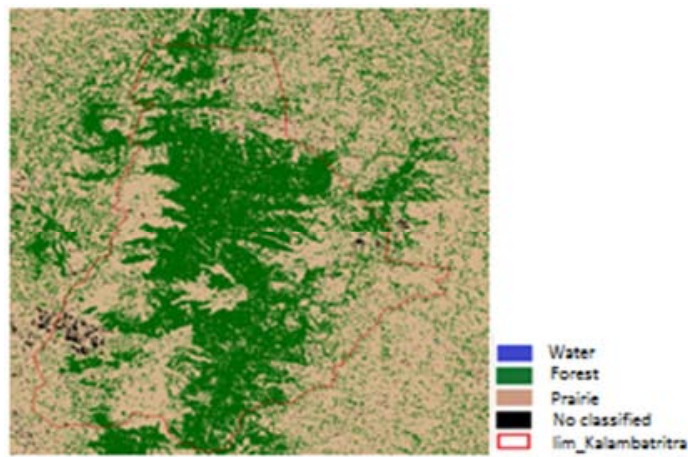

Figure 6. Result of the Kalambatritra classification with the SCM algorithm.

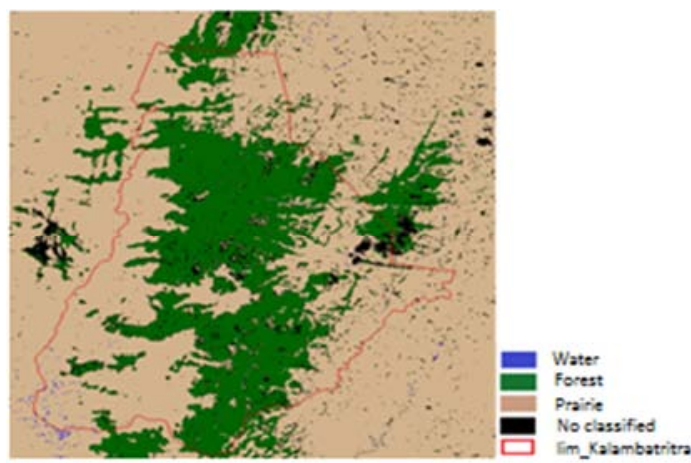

Figure7. Result of the Kalambatritra classification with the Maximum Likelihood Algorithm.

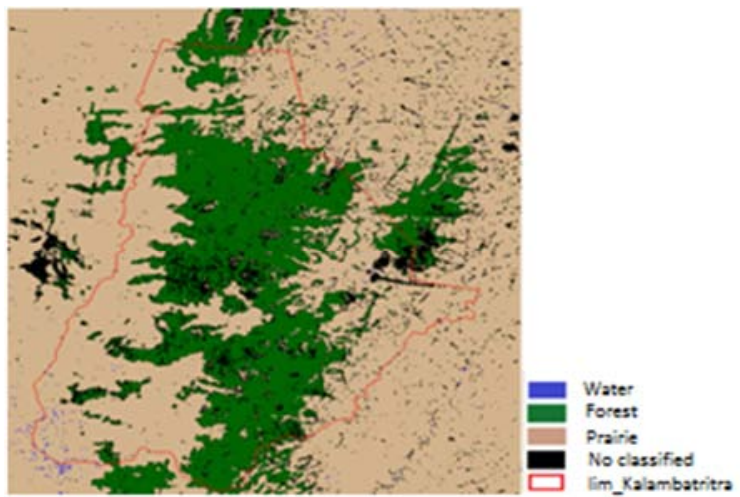

Figure 8. Result of the Kalambatritra classification with the Mahalanobis distance algorithm.

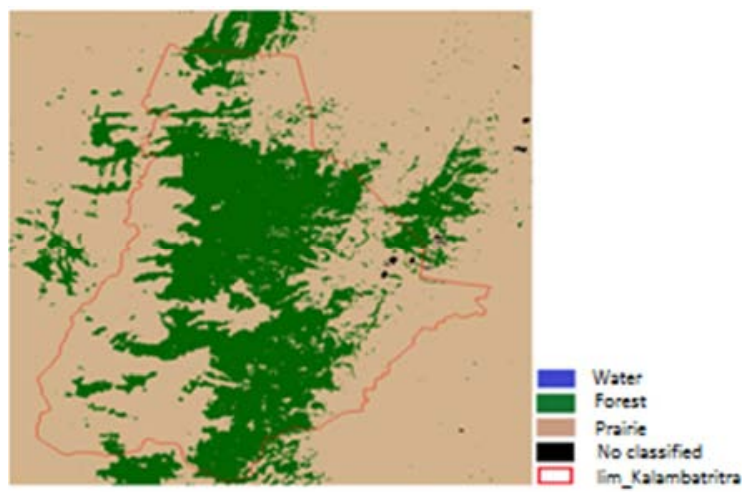

Figure 9. Result of the Kalambatritra classification with the minimum distance algorithm. 
After validation, each classification result is associated by a confusion matrix. Tables 2, 3, 4, 5, 6 and 7 show these matrices.

Table 2. Result of the validation with the SAM algorithm.

\begin{tabular}{llllll}
\hline Class & Reference & $\begin{array}{l}\text { Nbr } \\
\text { classified }\end{array}$ & $\begin{array}{l}\text { Well } \\
\text { classified }\end{array}$ & $\begin{array}{l}\text { Producer } \\
\text { Accuracy }\end{array}$ & $\begin{array}{l}\text { User } \\
\text { Accuracy }\end{array}$ \\
\hline Water & 14 & 6 & 6 & $42.86 \%$ & $100 \%$ \\
Forest & 38 & 33 & 33 & $86.84 \%$ & $100 \%$ \\
No classified & 3 & 13 & 3 & $100 \%$ & $23.08 \%$ \\
Prairie & 45 & 48 & 35 & $77.78 \%$ & $72.92 \%$ \\
\hline
\end{tabular}

Table 3. Result of the validation with the SCM algorithm.

\begin{tabular}{llllll}
\hline Class & Reference & $\begin{array}{l}\text { Nbr } \\
\text { classified }\end{array}$ & $\begin{array}{l}\text { Well } \\
\text { classified }\end{array}$ & $\begin{array}{l}\text { Producer } \\
\text { Accuracy }\end{array}$ & $\begin{array}{l}\text { User } \\
\text { Accuracy }\end{array}$ \\
\hline Water & 14 & 8 & 8 & $57.14 \%$ & $100 \%$ \\
Forest & 32 & 40 & 32 & $100 \%$ & $80.00 \%$ \\
No classified & 1 & 13 & 1 & $100 \%$ & $7.69 \%$ \\
Prairie & 53 & 39 & 34 & $64.15 \%$ & $87.18 \%$ \\
\hline
\end{tabular}

Table4. Result of the validation with the Maximum Likelihood algorithm.

\begin{tabular}{llllll}
\hline Class & Reference & $\begin{array}{l}\text { Nbr } \\
\text { classified }\end{array}$ & $\begin{array}{l}\text { Well } \\
\text { classified }\end{array}$ & $\begin{array}{l}\text { Producer } \\
\text { Accuracy }\end{array}$ & $\begin{array}{l}\text { User } \\
\text { Accuracy }\end{array}$ \\
\hline Water & 14 & 7 & 7 & $50 \%$ & $100 \%$ \\
Forest & 39 & 38 & 36 & $92.31 \%$ & $94.74 \%$ \\
No classified & 3 & 3 & 3 & $100 \%$ & $100 \%$ \\
Prairie & 43 & 52 & 42 & $97.67 \%$ & $80.77 \%$ \\
\hline
\end{tabular}

Table 5. Result of the validation with the Mahalanobis Distance Algorithm.

\begin{tabular}{llllll}
\hline Class & Reference & $\begin{array}{l}\text { Nbr } \\
\text { classified }\end{array}$ & $\begin{array}{l}\text { Well } \\
\text { classified }\end{array}$ & $\begin{array}{l}\text { Producer } \\
\text { Accuracy }\end{array}$ & $\begin{array}{l}\text { User } \\
\text { Accuracy }\end{array}$ \\
\hline Water & 14 & 6 & 6 & $42.86 \%$ & $100.00 \%$ \\
Forest & 37 & 33 & 32 & $86.49 \%$ & $96.97 \%$ \\
No classified & 10 & 15 & 10 & $100.00 \%$ & $66.67 \%$ \\
Prairie & 39 & 46 & 36 & $92.31 \%$ & $78.26 \%$ \\
\hline
\end{tabular}

Table6. Result of the validation with the algorithm Minimum distance.

\begin{tabular}{llllll}
\hline Class & Reference & $\begin{array}{l}\text { Nbr } \\
\text { classified }\end{array}$ & $\begin{array}{l}\text { Well } \\
\text { classified }\end{array}$ & $\begin{array}{l}\text { Producer } \\
\text { Accuracy }\end{array}$ & $\begin{array}{l}\text { User } \\
\text { Accuracy }\end{array}$ \\
\hline Water & 15 & 7 & 7 & $42.67 \%$ & $100 \%$ \\
Forest & 38 & 36 & 36 & $94.74 \%$ & $100 \%$ \\
No classified & 6 & 6 & 6 & $100 \%$ & $100 \%$ \\
Prairie & 41 & 51 & 41 & $100 \%$ & $80.39 \%$ \\
\hline
\end{tabular}

Table 7. Comparison of results for each classifier.

\begin{tabular}{lll}
\hline classifier & $\begin{array}{l}\text { Overall Classification } \\
\text { Accuracy }\end{array}$ & $\begin{array}{l}\text { Overall Kappa } \\
\text { Statistics }\end{array}$ \\
\hline SAM & $77 \%$ & 0.64 \\
SCM & $75 \%$ & 0.6170 \\
ML & $88 \%$ & 0.8057 \\
D Mahalanobis & $84 \%$ & 0.7630 \\
D minimum & $90 \%$ & 0.84 \\
\hline
\end{tabular}

Figure 10 shows a graphical representation in histogram form of the variation in the results obtained during the treatments.
Table 8. Result of Kalambatritra Area Calculations.

\begin{tabular}{ll}
\hline Object & Area(ha) \\
\hline Water & - \\
Forest & 13462,1842 \\
No classified & 38,1336642 \\
Prairie & 16798,8006 \\
\hline
\end{tabular}

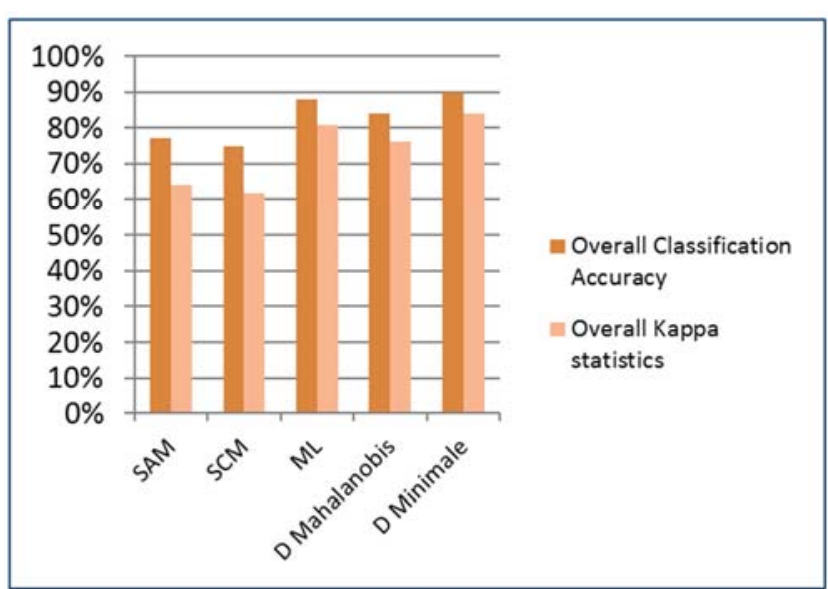

Figure 10. Histogram representation of the validation results.

Figure11 shows us a mapping of the result of the classification using the distance algorithm.

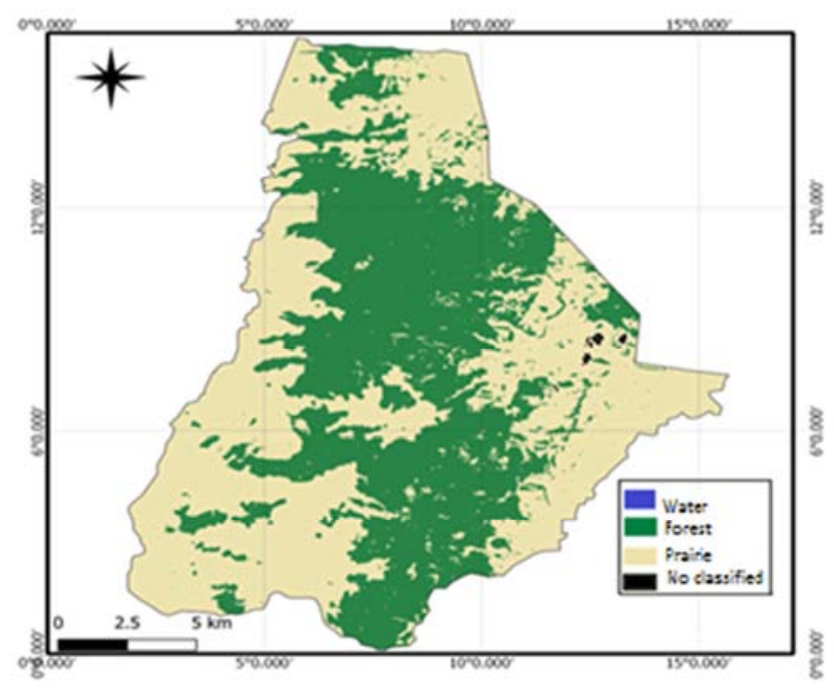

Figure 11. Map showing the forest area of Kalambatritra in 2018.

Table 8 shows the area of Forest, Prairie and Unclassified of Kalambatritrain 2018.

\section{Discussions}

This study allowed us to determine the forest area for the year 2018. Moreover, we could not find the evolution of the Kalambatritra forest area between years. This limitation recommends us to use other previous images or images at different dates to detect the change existing in this area using the change detection algorithm.

The resolution of an image is very important in a supervised classification especially in visual analysis. An image with low 
resolution hinders forest type categorization. However, we only have a resolution of $15 \mathrm{~m}$ for our multispectral image after fusion. This resolution will not allow the categorization of the Kalambatritra forests. Thus, this opens the door to the fusion of Landsat with other very high spatial resolution satellite images such as SPOT, Sentine 1.

During the experiments we have done, our treatments are notably manual and repetitive. In addition, we spent a lot of time. The automation of the processing will be so interesting to improve the work organization, optimize the execution time and make easy the tedious work in satellite image processing. Thus, the use of libraries like OTB, R will allow this automation.

For these five supervised classification algorithms, their processing times and their algorithm result accuracies are relatively different. The Spectral Correlation Mapper was very slow compared to the other algorithms while the minimum distance gave the smallest processing time but with the highest accuracy. Thus, we can conclude that the classification algorithm plays a very important role in terms of result accuracy and processing time optimization.

Comparing our result of comparison of classification algorithms to another result of a paper [20], our finding is different to its result in terms of precision and qualification rank because this paper obtained the highest precision in maximum likelihood algorithm, then the SAM, the SCM while the minimum distance gave the worst result. This differentiation is normal taking into account the type of data used, the characteristic of the study area as well as the number of classes.

The presence of clouds on the image to be processed makes it particularly difficult to classify the Kalambatritra area because this special reserve has white layers that cover certain stones. In addition, the Kalambatritra special reserve is crossed by a stream. It is difficult to classify this brook with a resolution of $15 \mathrm{~m}$ the fact that it is confused with the characteristic of the Prairie class. Thus, the Kalambatritra classification requires the use of a very powerful classification algorithm capable of distinguishing the spectral signature between objects.

\section{Conclusion and Perspectives}

In this study, we performed the supervised classification of the Kalambatritra Special Reserve from a Landsat 8 satellite image of OLI sensor year 2018. For this, we compared the five algorithms of ERDAS Imagine. After validating the results obtained, we observed that the minimum distance algorithm gave the highest classification accuracy for Kalambatritra with 90\% Overall Classification Accuracy and 0.84 Kappa index. This result led us to the production of a mapping and land cover area calculation for Kalambatritra.

Perspectives include the use of multidate images at change detection to produce maps of change in Kalambatritra forest area, the use of multi-source images (SPOT, Sentinel) to categorize Kalambatritra forests. We also envisage the use of OTB, R, Python libraries to automate processing as well as the introduction on machine learning algorithms and especially the use of deep learning architectures. These will be able to give some observations and improvements on the accuracy of the classification result.

\section{References}

[1] Aimé Richard Hajalalaina, Manuel Grizonnet, Eric Delaitre, Solofo Rakotondraompiana, Dominique Hervé (2013); Discrimination des zones humides en foret Malgache, proposition d'une methodologie multiresolution et multisource utilisant orfeo toolbox;Revue de Photogrammetrie et de télédétection.

[2] Avisoatolona Andrianarivo, Eric Delaitre, Anne Elisabeth Laques, Stéphanie. M. Carriere, Dominique Hervé (2015); Détection de changement de l'occupation du sol dans une commune à la peripherie de la forêt humide de Fianarantsoa;

[3] Nadia OUARAB, Youcef SMARA, Jean-Paul RASSON (1999); Utilisation de methods de classification hierarchique pour une classification supervisée d'image; Dix-septième colloque GRETSI, Vannes;

[4] Marine Campedel, Eric Moulines (2014); Méthodologie de sélection de caractéristiques pour la classification d'images satellitaires;

[5] Salim CHITROUB (2004); Combinaison de classiffeurs:une approche pour l'amelioration de la classification d'images multisources/Multidates de teledetection; Télédétection,, vol. 4, n³, p. 289-301;

[6] Aimé Richard Hajalalaina, Dominique Hervé, J. P Razafimandimby, E. Delaitre, J. C. Desconnets, T. Libourel (2016); Formalisation des chaines de traitements de données spatiales satellitaires sur la forêt à Madagascar;

[7] Rashmi S, Swapna Addamani, Venkat and Ravikiran S (2014); Spectral Angle Mapper Algorithm for Remote Sensing Image Classification; ISSN 2348 - 7968; Vol. 1 Issue 4;

[8] Bertels Luc, Bart Deronde, Pieter Kempeneers, Walter Debruyn, Sam Provoost (2005); Optimized Spectral Angle Mapper classification of spatially heterogeneous dynamic dune vegetation, a case study along the Belgian coastiline; The $9^{\text {th }}$ international Symposium on Physical Measurements and Signatures in Remote Sensing (ISPMSRS). Beijing, October 17-19.

[9] George P. Petropoulos, Krishna Prasad Vadrevu \& Chariton Kalaitzidis (2012): Spectral angle mapper and object-based classification combined with hyperspectral remote sensing imagery for obtaining land use/cover mapping in a Mediterranean region, Geocarto International, DOI: $10.1080 / 10106049.2012 .668950$

[10] Kruse, F. A., et al., 1993. The spectral image processing system (sips) - interactive visualization and analysis of imaging spectrometer data. Remote Sensing of Environment, 44, 145163.

[11] Keerti Kulkarni, P. A. Vijaya (2019); Experiment of Multispectral Images usin Spectral Angle Mapper Algorithm for Land; International Journal of Innovative Technology and Exploring Engineering (IJITEE); ISSN: 2278-3075, Volume-8, Issue-6S4, April 2019. 
[12] Osmar Abílio de Carvalho Jr, Paulo Roberto Meneses; Spectral Correlation Mapper (SCM): An Improvement on the Spectral Angle Mapper (2000).

[13] Natanael Antunus Abade, Osmar Abilio de Carvalho Junior, Renato Fontes Guimaraes, Sandro Nunes de Oliveira (2005); Comparative Analysis of Modis Time-Series Classification Using Support Vector Machines and Methods Based upon Distance and Similarity Measures in the Brazilian Cerrado-Caatinga Boundary; remote sensing ISSN 2072-4292;

[14] Madhura M, Suganthi Venkatazchalam (2013); Comparison of Supervised Classification Methods on Remode Sensed Satellite Data:An Application In Chennai, South India; International Journal of Science and Research (IJSR); ISSN: 2319-7064;

[15] F. S. Al-Ahmadi, A. S. Hames (2008); Comparaison of four classification Methods to Extract Land Use and Land Cover from Raw Satellite Images for Remote Arid Areas, Kingdom of Saudi Arabia; JKAU; Earth Sci., Vol. 20 No, 1, pp: 167-191 (2009 A.D./1430 A.H);
[16] Mahendra H N, Mallikarjunaswamy S, Rekha V, Puspalatha V, Sharmila N(2019); Performance Analysis of Different Classifier for Remote Sensing Application; International Journal of Engineering and Advanced Technology (IJEAT) ISSN: 2249-8958, Volume-9 Issue-1;

[17] Bahadir Durak (2011); A classification Algorithme using Mahalanobis Distance clustering of data with applications;

[18] Umair Khan, Nasru Minallah, Ahmad Junaid, Kashaf Gul, Nasir Ahmad (2015); Parallelepiped and Mahalanobis Distance based Classification for forestry identification in Pakistan; IEEE 978-1-5090-0436-2

[19] Ameur Zohra, Adane Abdelhamid, Ameur Soltane (2007); Etude comparative des méthodes de classification appliquée à la segmentation d'images texturées;

[20] J. H. Lumme (2004), Classification of vegetation and soil using imaging spectrometer data; Geoscience and remote sensing symposium; 